\title{
Atomic Structure of Intrinsic and Electron-Irradiation-Induced Defects in $\mathrm{MoTe}_{2}$
}

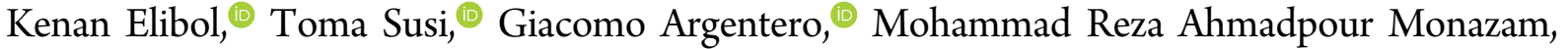 \\ Timothy J. Pennycook, Jannik C. Meyer, ${ }^{\odot}$ and Jani Kotakoski*º \\ Faculty of Physics, University of Vienna, Boltzmanngasse 5, A-1090 Vienna, Austria
}

\section{Supporting Information}

\begin{abstract}
Studying the atomic structure of intrinsic defects in two-dimensional transition-metal dichalcogenides is difficult since they damage quickly under the intense electron irradiation in transmission electron microscopy (TEM). However, this can also lead to insights into the creation of defects and their atom-scale dynamics. We first show that $\mathrm{MoTe}_{2}$ monolayers without protection indeed quickly degrade during scanning TEM (STEM) imaging, and discuss the observed atomic-level dynamics, including a transformation from the $1 \mathrm{H}$ phase into $1 \mathrm{~T}^{\prime}, 3$-fold rotationally

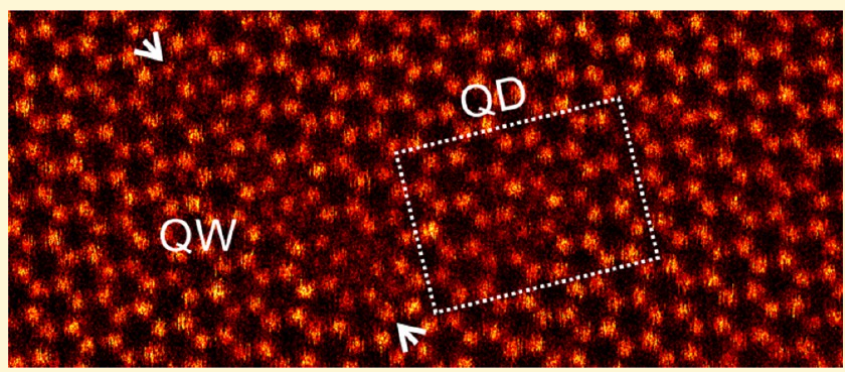
symmetric defects, and the migration of line defects between two $1 \mathrm{H}$ grains with a $60^{\circ}$ misorientation. We then analyze the atomic structure of $\mathrm{MoTe}_{2}$ encapsulated between two graphene sheets to mitigate damage, finding the as-prepared material to contain an unexpectedly large concentration of defects. These include similar point defects (or quantum dots, QDs) as those created in the nonencapsulated material and two different types of line defects (or quantum wires, QWs) that can be transformed from one to the other under electron irradiation. Our density functional theory simulations indicate that the QDs and QWs embedded in $\mathrm{MoTe}_{2}$ introduce new midgap states into the semiconducting material and may thus be used to control its electronic and optical properties. Finally, the edge of the encapsulated material appears amorphous, possibly due to the pressure caused by the encapsulation.
\end{abstract}

\section{INTRODUCTION}

Knowing the atomic arrangement of materials beyond the crystal structure is imperative for understanding their real-life performance, as defects often have a profound influence on material properties. The recent combination of advances in aberration corrected transmission electron microscopy (TEM) and two-dimensional (2D) materials has provided an unprecedented possibility both to directly image defects and to control them. ${ }^{1}$ Among 2D materials, the family of transitionmetal dichalcogenides (TMDC) with the chemical structure $\mathrm{MX}_{2}$ ( $\mathrm{M}$ is a transition metal and $\mathrm{X}$ is a chalcogen atom) displays arguably the widest range of material properties. Remarkably, even the same TMDC in its different configurations can exhibit different behaviors. For example, $2 \mathrm{H}, 1 \mathrm{~T}$, $1 \mathrm{~T}^{\prime}$, and $\mathrm{T}_{d}$ phases of $\mathrm{MoTe}_{2}$ all have different electronic characteristics (semiconductor, metal, semimetal, and superconductor, respectively). ${ }^{2-5}$ Also the electron mobilities of the different phases vary: $1 \mathrm{~T}^{\prime}$ has a mobility of $4000 \mathrm{~cm}^{2} /(\mathrm{V} \mathrm{s})$, whereas that of $2 \mathrm{H}$ is 2 orders of magnitude lower. ${ }^{6}$ Further, similar to other TMDCs, although monolayer $\mathrm{MoTe}_{2}$ is a direct bandgap semiconductor, its bulk form has an indirect bandgap. ${ }^{7}$

Of the different $\mathrm{MoTe}_{2}$ phases, the normally metastable $1 \mathrm{~T}^{\prime}$ becomes stable under strain against the otherwise more stable $2 \mathrm{H}^{8,9}$ At temperatures below $240 \mathrm{~K}, 1 \mathrm{~T}^{\prime}$ is expected to transition into the superconducting $\mathrm{T}_{d}$ phase. ${ }^{10}$ Until now, different phases have been obtained in $\mathrm{MoTe}_{2}$ through methods including strain and exposure to electron beams, light, and different temperatures. ${ }^{11-15}$ However, the degree of control regarding the location and size of the transformed regions has remained poor.

Defects such as vacancies, grain boundaries, and edges have a significant impact on the physical properties of TMDCs. For example, it is possible to induce n-type or p-type transport in $\mathrm{MoS}_{2}$ via sulfur or molybdenum vacancies. ${ }^{16}$ Looped line defects consisting of 8-5-5-8-membered rings in $\mathrm{MoS}_{2}$ and $\mathrm{WSe}_{2}$ induce midgap states, ${ }^{17-19}$ as do grain boundaries consisting of 8-4-4- or 8-4-4-8-membered rings in $\mathrm{MoS}_{2}{ }^{20,21}$ Mirror-symmetric grain boundaries in $\mathrm{MoSe}_{2}$ and $\mathrm{MoTe}_{2}$, studied both theoretically and experimentally by scanning tunneling spectroscopy, also show localized states within the band gap, ${ }^{10,22,23}$ in contrast to the mirror-symmetric grain boundary in $\mathrm{MoS}_{2}$ which acts as a $1 \mathrm{D}$ metallic quantum wire. ${ }^{24}$ Similarly, the optical properties of TMDCs are altered by defects because the response is directly related to the electronic band structure. ${ }^{25}$ The edges of TMDCs also have attractive optical properties since the intensity of visible light emitted by them is as high or even higher than the entire bulk of the crystal. ${ }^{16}$ Further, dopants in TMDCs induce localized states in

Received: September 5, 2017

Revised: February 5, 2018

Published: February 5, 2018 


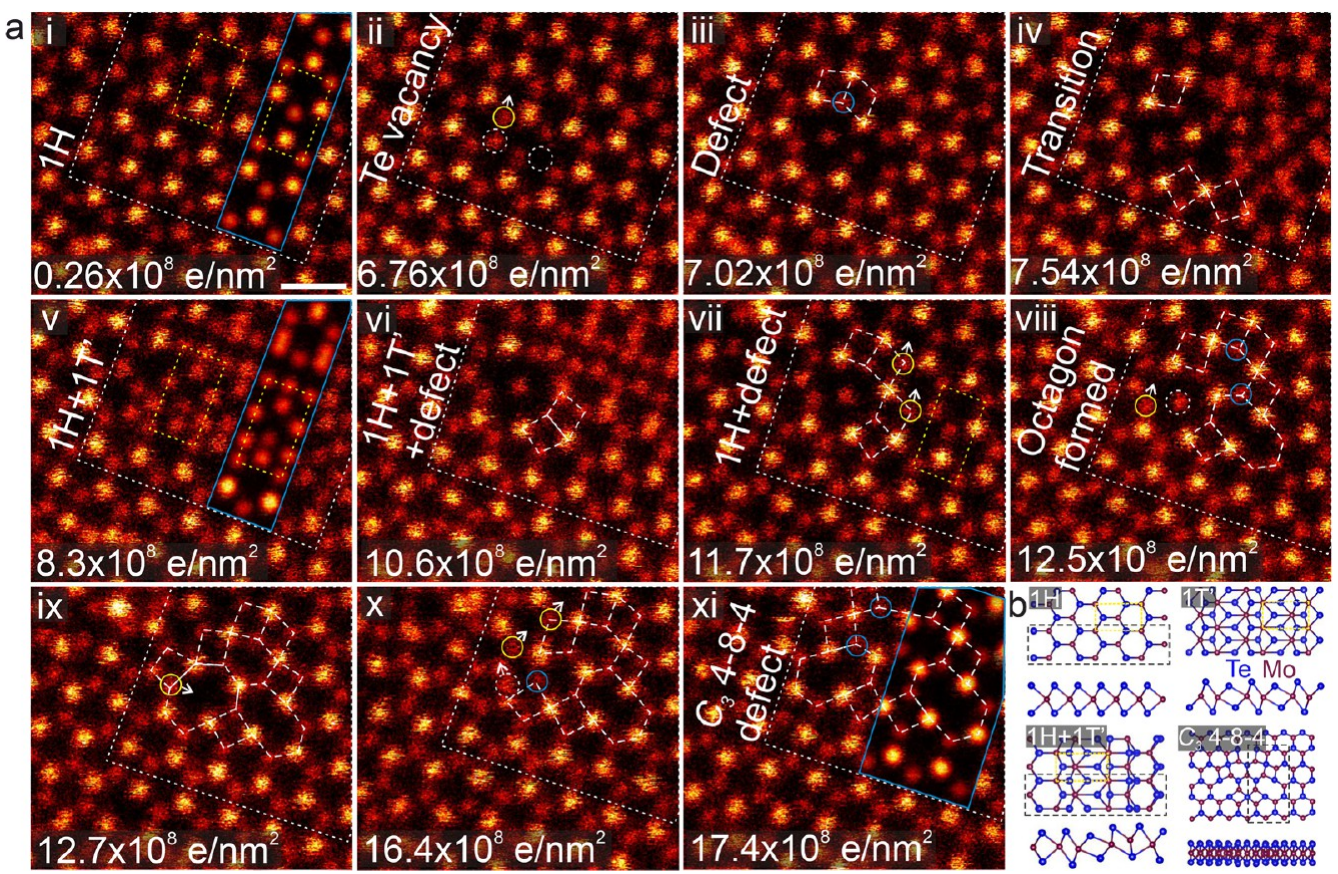

Figure 1. Phase transition and a 3-fold rotationally symmetric defect in a $\mathrm{MoTe}_{2}$ monolayer. (a) STEM-HAADF image sequence showing two phase transitions $\left(1 \mathrm{H} \rightarrow 1 \mathrm{~T}^{\prime}\right.$ and $\left.1 \mathrm{~T}^{\prime} \rightarrow 1 \mathrm{H}\right)$ under electron irradiation. The overlaid areas outlined by blue lines in panels a-i, a-v, and a-xi are simulated images using the black dashed regions of the models in panel b. Images without overlays are shown in Supplementary Figure 2. The unit cells of the $1 \mathrm{H}$ and $1 \mathrm{~T}^{\prime}$ phases are marked by yellow dashed frames. Te vacancies are marked by white dashed circles, while the Mo atom, before and after it shifts, is marked with yellow and blue solid circles, respectively. The areas surrounded by the white dashed lines show the same region in all frames. The experimental images display raw data in false color, and the scale bar is $0.5 \mathrm{~nm}$.
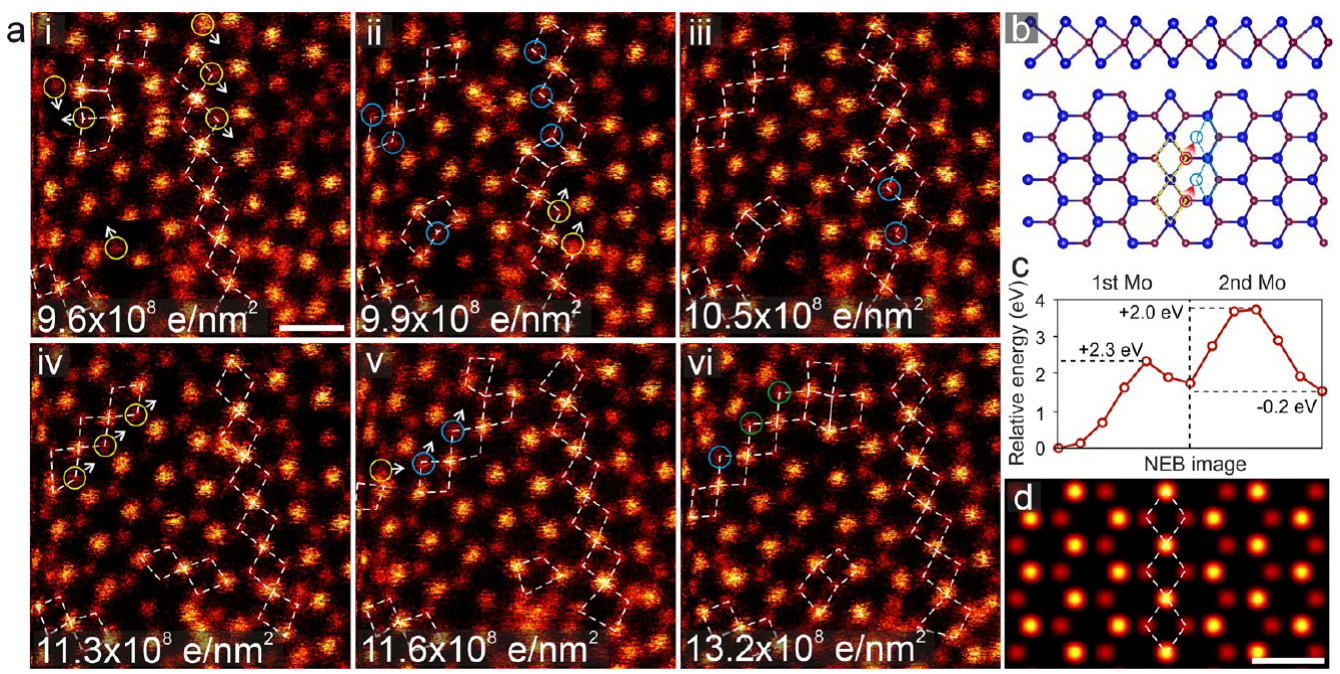

Figure 2. Migration of grain boundaries in a $\mathrm{MoTe}_{2}$ monolayer. (a) STEM-HAADF image sequence showing the migration of grain boundaries. The arrows on the images show the direction of movement of the Mo atoms marked with circles. (b) Simulated model of the grain boundary. The migration of the boundary atoms is schematically indicated with arrows, similar to the experimental images. (c) Migration barriers for two of the Mo atoms at a four-unit model of the grain boundary calculated via DFT using the nudged elastic band method. (d) Image simulation of the grain boundary model. The experimental images display raw data in false color, and the scale bars are $0.5 \mathrm{~nm}$.

the electronic band structure and also shift the photoluminescence energy. ${ }^{16}$ Recent studies demonstrate that producing atomic vacancies via electron- or ion-beam irradiation can enhance ferromagnetism in $\mathrm{MoS}_{2}{ }^{16}$ and that grain boundaries consisting of 5-7 dislocation cores exhibit ferromagnetic behavior whereas those with 4-8 dislocation cores are antiferromagnetic. ${ }^{16,26}$ Despite such efforts toward finding new physical properties and functionalities in various
$2 \mathrm{D}$ materials, the atomic structure of defects in $\mathrm{MoTe}_{2}$ remains poorly understood.

In this work, we study the atomic structure of $\mathrm{MoTe}_{2}$ samples consisting of mechanically exfoliated mono-, bi-, and triple-layer areas with scanning transmission electron microscopy (STEM). Although the as-exfoliated material deteriorates quickly under electron irradiation due to ionization damage, we were able to image local phase transitions between the $1 \mathrm{H}$ and $1 \mathrm{~T}^{\prime}$ phases and the formation of 3-fold rotationally symmetric 

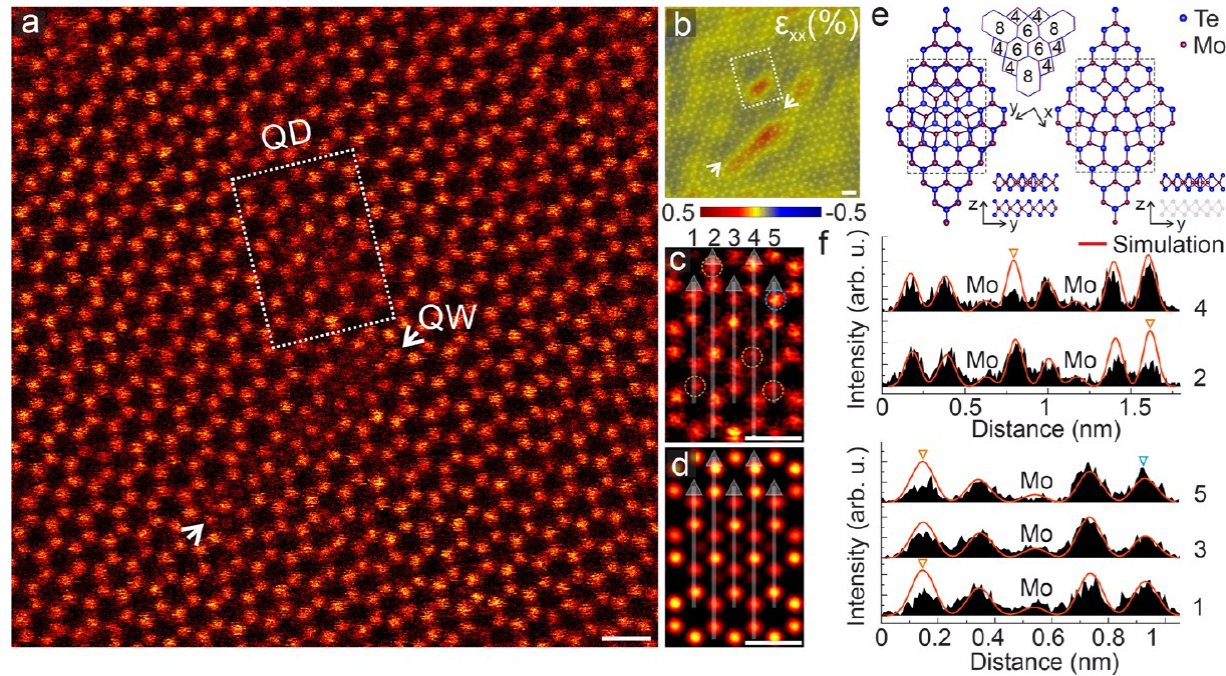

Figure 3. Structure of defects in encapsulated bilayer $\mathrm{MoTe}_{2}$. (a) STEM-HAADF image of an encapsulated MoTe $\mathrm{M}_{2}$ bilayer with a QD defect (white dashed rectangle) and QW (marked by arrows). Image background has been subtracted using a Gaussian blur with a radius of 20 px. (b) $\varepsilon_{x x}$ strain map of panel a overlaid on the atomic structure. (c) A close-up of the QD (treated by a Gaussian blur with a radius of 1 px). Orange and blue dashed circles show atomic vacancies and additional atoms, respectively. (d) Image simulation for the atomic model shown in panel e. (e) QD model with and without the second $\mathrm{MoTe}_{2}$ layer. Top views of the $\mathrm{QD}$ atomic model with a 3-fold rotationally symmetric defect consisting of 4-8-4-membered rings in the upper $\mathrm{MoTe}_{2}$ layer. (f) Intensity profiles along the white semitransparent arrows shown in panels $\mathrm{c}$ and $\mathrm{d}$. Triangles show the locations of atomic vacancies and additional atoms as indicated in panel c. The scale bars are $0.5 \mathrm{~nm}$.

defects consisting of 4-8-4-membered rings at high electron doses, as well as the migration of grain boundaries between areas with opposite crystalline orientations $(1 \mathrm{H}$ grains with misorientation of $60^{\circ}$ ). For further studies, we encapsulated $\mathrm{MoTe}_{2}$ between two graphene monolayers to both protect the material from oxidation and to mitigate damage during STEM imaging. We show that the as-prepared samples contain 3-fold rotationally symmetric quantum dots (QDs), and both reflection symmetric (QW1) and 2-fold rotational symmetric (QW2) quantum wires (QWs), all of which show dynamics during the experiment. We also find that the edge of the encapsulated material appears amorphous and changes constantly under the electron beam, which complicates the identification of the elemental structure of the edge.

\section{RESULTS}

Atomic Scale Dynamics in Free-Standing $\mathrm{MoTe}_{2}$. As with other TMDCs, ${ }^{27-29} \mathrm{MoTe}_{2}$ monolayers damage quickly under electron irradiation (see Supplementary Figure 1). Nevertheless, it is possible to obtain atomic-resolution images of the structure when the exposure of the material is minimized. In Figure 1a we show an image sequence displaying initially a nearly perfect $1 \mathrm{H}$ structure, which is then partially converted into a $1 \mathrm{~T}^{\prime}$ phase in panel a-v (after an additional electron dose of $\left.\sim 8.1 \times 10^{8} e^{-} / \mathrm{nm}^{2}\right)$. The energy difference between $1 \mathrm{H}$ and $1 \mathrm{~T}^{\prime}$ is just $0.03 \mathrm{eV} /$ atom (see Supplementary Table 1) at zero pressure, and $1 \mathrm{~T}^{\prime}$ becomes favored under strains between $0.3 \%$ and $3 \%$ at room temperature. ${ }^{9}$ Hence, the local strain induced by the initially created Te vacancies (at a dose of $\sim 6.8 \times 10^{8}$ $e^{-} / \mathrm{nm}^{2}$ ) most likely serves as the driving force for the observed transition from $1 \mathrm{H}$ to $1 \mathrm{~T}^{\prime}$. After the vacancies have been created, some Mo atoms close to the defect shift to create diamond-shaped 4-membered rings. This leads eventually to the creation of a mixed $1 \mathrm{H}-1 \mathrm{~T}^{\prime}$ area (after a dose of $\sim 8.3 \times 10^{8}$ $e^{-} / \mathrm{nm}^{2}$ ). Further exposure to electron irradiation leads first to the creation of vacancies in the $1 \mathrm{~T}^{\prime}$ phase and finally to its disappearance. Afterward, only the defected $1 \mathrm{H}$ phase remains.
After a dose of $\sim 12.5 \times 10^{8} e^{-} / \mathrm{nm}^{2}$, an 8 -membered ring appears in addition to the diamond-shaped ones. At the end of the image sequence, a structure consistent with a 3-fold rotationally symmetric $\left(C_{3}\right)$ defect made of 4-8-4-membered rings has been created.

In another image sequence (Figure 2), we show a mirrorsymmetric grain boundary between two $1 \mathrm{H}$ grains at $\sim 60^{\circ}$ misorientation, similar to what has been reported in $\mathrm{MoS}_{2}$, $\mathrm{MoSe}_{2}$, and $\mathrm{MoTe}_{2} / \mathrm{MoS}_{2}$ heterostructures. ${ }^{22-24,30}$ At least in our case, the area around the boundary was initially a singlecrystalline area of $1 \mathrm{H}$, and the misoriented grains only appeared during the experiment (see also Supplementary Figures 3, 4, and 5). During the image sequence (Figure 2), molybdenum atoms at the grain boundary (typically) undergo correlated migration by half a lattice vector (see for example the three atoms marked yellow in Figure 2a-i where the arrow indicates the direction of movement; the new positions are marked in panel a-ii with blue circles), allowing the migration of the grain boundary within the crystal. According to our density functional theory (DFT) simulations (Methods), the migration of the first Mo atom at the boundary has an energy barrier of $2.3 \mathrm{eV}$, dropping to $2.0 \mathrm{eV}$ for the second one. The migration of the mirror-symmetric grain boundaries in $\mathrm{MoTe}_{2}$ starts with the displacement of Mo atoms by half a lattice vector, which is similar to the grain boundary migration in $\mathrm{MoSe}_{2}{ }^{31}$ In both materials, chalcogen vacancies are formed in the lattice by electron irradiation before Mo atoms shift to stabilize the system. However, for the grain boundaries in $\mathrm{WSe}_{2}{ }^{17}$ and $\mathrm{WS}_{2}{ }^{32}$ the migration starts with the movements of chalcogen atoms around the dislocation core in contrast to $\mathrm{MoTe}_{2}$ and $\mathrm{MoSe}_{2}$.

Quantum Dots. One of the most commonly observed defects in our samples is a QD structure shown for an encapsulated bilayer in Figure 3 (graphene is not visible due to the low contrast of $\mathrm{C}$ atoms as compared to Mo and Te; see also Supplementary Figure 6). We observed a concentration of $0.58 / 100 \mathrm{~nm}^{2}$ for the QDs (total area of $9500 \mathrm{~nm}^{2}$ was 


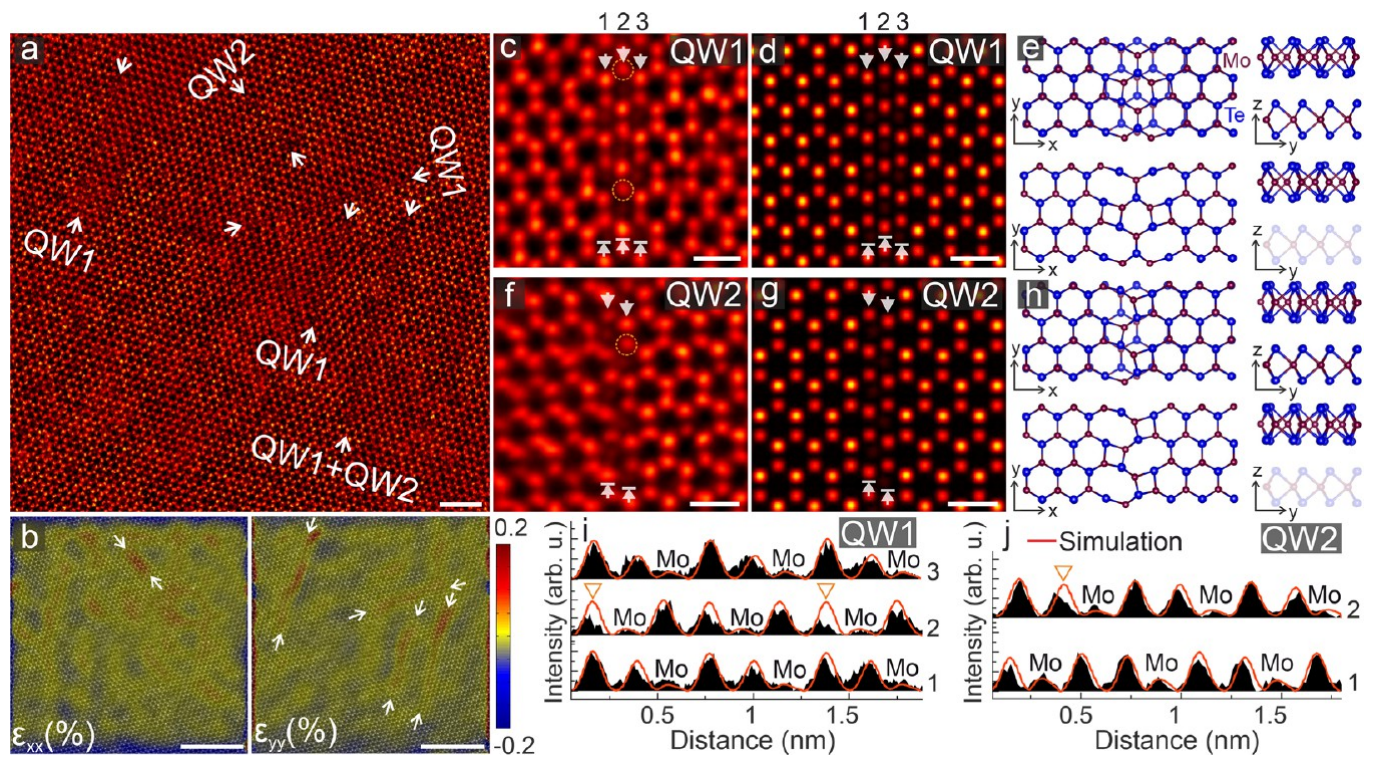

Figure 4. Atomic structure of quantum wires. (a) STEM-HAADF image of several QWs in encapsulated bilayer MoTe $\mathrm{C}_{2}$. Image background has been subtracted using a Gaussian blur with a radius of $12 \mathrm{px}$. The scale bar is $2 \mathrm{~nm}$. (b) $\varepsilon_{x x}$ and $\varepsilon_{y y}$ strain maps of panel (a). The scale bar is $4 \mathrm{~nm}$. (c,f) Close-up images of the two different QW structures (treated by a Gaussian blur with a radius of $3 \mathrm{px}$ ). Orange dashed circles show atomic vacancies. $(\mathrm{d}, \mathrm{g})$ Simulated images corresponding to the experimental structures. The scale bars are $0.5 \mathrm{~nm}$. (e,h) Atomic models of the simulated images (top: both layers, bottom: only the defective layer). (i,j) Intensity profiles along the white arrows on the experimental and simulated QW images. Triangles show the locations of atomic vacancies as indicated in panels (c) and ( $\mathrm{f})$.

imaged). This defect appears as an ca. $1 \mathrm{~nm}$ round feature within the $2 \mathrm{H}$ phase. Because of the different atomic structure with respect to the surrounding crystal, the QD can also be visualized through geometric phase analysis (along with the resulting strain maps, see Figure $3 \mathrm{~b}$ ). Within the QD, the atomic structure has the appearance of the $1 \mathrm{~T}$ phase. To ascertain the actual atomic structure, we created a large number of candidate structures for the defects and simulated STEM images based on DFT-relaxed atomic configurations. The best match to the experimental images was obtained for the structure shown in Figure 3. In this structure, one of the layers of the bilayer $\mathrm{MoTe}_{2}$ involves a 3-fold rotationally symmetric defect consisting of 4-8-4-membered rings, whereas the other layer is pristine. This defect is similar to that found in the nonencapsulated material after extensive electron-beam irradiation (Figure 1a-xi). An atomic model of the defected layer is shown in panel e. Intensity profiles recorded on the white lines over the simulated (panel $d$ ) and experimental images (panel c) show that the atomic model and the experimental image are in a good agreement. The intensity profiles also reveal that there are $\mathrm{Te}$ vacancies and one additional atom in this experimental QD structure as compared to the model (such irregularities, appearing at different atoms for each defect, are common among all the QD structures we observed). Unlike defects created by electron irradiation, these defects in one layer of the encapsulated $\mathrm{MoTe}_{2}$ bilayer are likely to have been formed during sample growth or preparation since they were observed from the beginning of the imaging and formation of additional QDs was only very rarely observed during the experiments.

Quantum Wires. Besides the QD structures, our encapsulated samples also contain two different kinds of QWs (one was visible in Figure 3a). Several examples of both are shown in Figure 4. The concentration of QWs (0.48/100 $\mathrm{nm}^{2}$ in a total observed area of $9500 \mathrm{~nm}^{2}$ ) is slightly lower than that of the QDs, and they can also be easily detected from microscopy images through strain maps (Figure 4b), either in $\varepsilon_{x x}$ or $\varepsilon_{y y}$ depending on the direction of the QW with respect to the host lattice. A close-up image of the first type (QW1) is shown in Figure $4 c$ along with a simulated image (Figure $4 d$ ) and the corresponding atomic structure (Figure 4e); QW2 is similarly displayed in Figure 4f-h. The atomic structures of the defects are shown in Figure 4e,h. QW1 includes reflection symmetric $\left(\sigma_{v} \|\right)$ units consisting of 8 -4-membered rings (Figure 4e). In contrast, QW2 is made of 2 -fold rotational symmetric units $\left(C_{2}\right)$ also consisting of 8-4-membered rings (Figure $4 \mathrm{~h}$ ). Line profiles over the experimental and simulated STEM-HAADF images are plotted in Figure 4i,j. Similarly to QDs, QW structures also include Te vacancies. QD and QW defects are less stable compared to pristine $1 \mathrm{H}-\mathrm{MoTe}_{2}$, and their formation energies are found to be $2.80,4.81$, and $3.73 \mathrm{eV}$ respectively for QD, QW1, and QW2 (see also Supplementary Table 2). Among these defect structures, QD has one more Mo atom than a pristine structure of the same size, whereas the QWs are stoichiometric. The formation energy of the QD has thus been calculated using the chemical potential for bulk molybdenum. Line defects in the armchair direction were previously reported for few-layer $\mathrm{MoTe}_{2} \cdot{ }^{33}$ In that work, it was concluded that line defects are mostly observed in only one of the $\mathrm{MoTe}_{2}$ layers within the few-layer structures, similar to the defects reported here. Further, it was suggested that line defects are formed via the rearrangement of Te vacancies. By contrast, the line defects discussed in our study are formed via the rearrangement of Mo atoms.

Evolution of QD and QW Structures during Observation. Figure 5 shows an image sequence of an area containing both a QD and a QW (initially having the QW2 structure). Strain maps corresponding to the image sequence in Figure 5a are shown in Figure 5b. Although the QW is barely visible in these strain maps due to its orientation, the $\mathrm{QD}$ is easily visible. After an additional electron dose of $\sim 0.4 \times 10^{8} e^{-} / \mathrm{nm}^{2}$, a vacancy defect appears next to the QD. At the same time, 

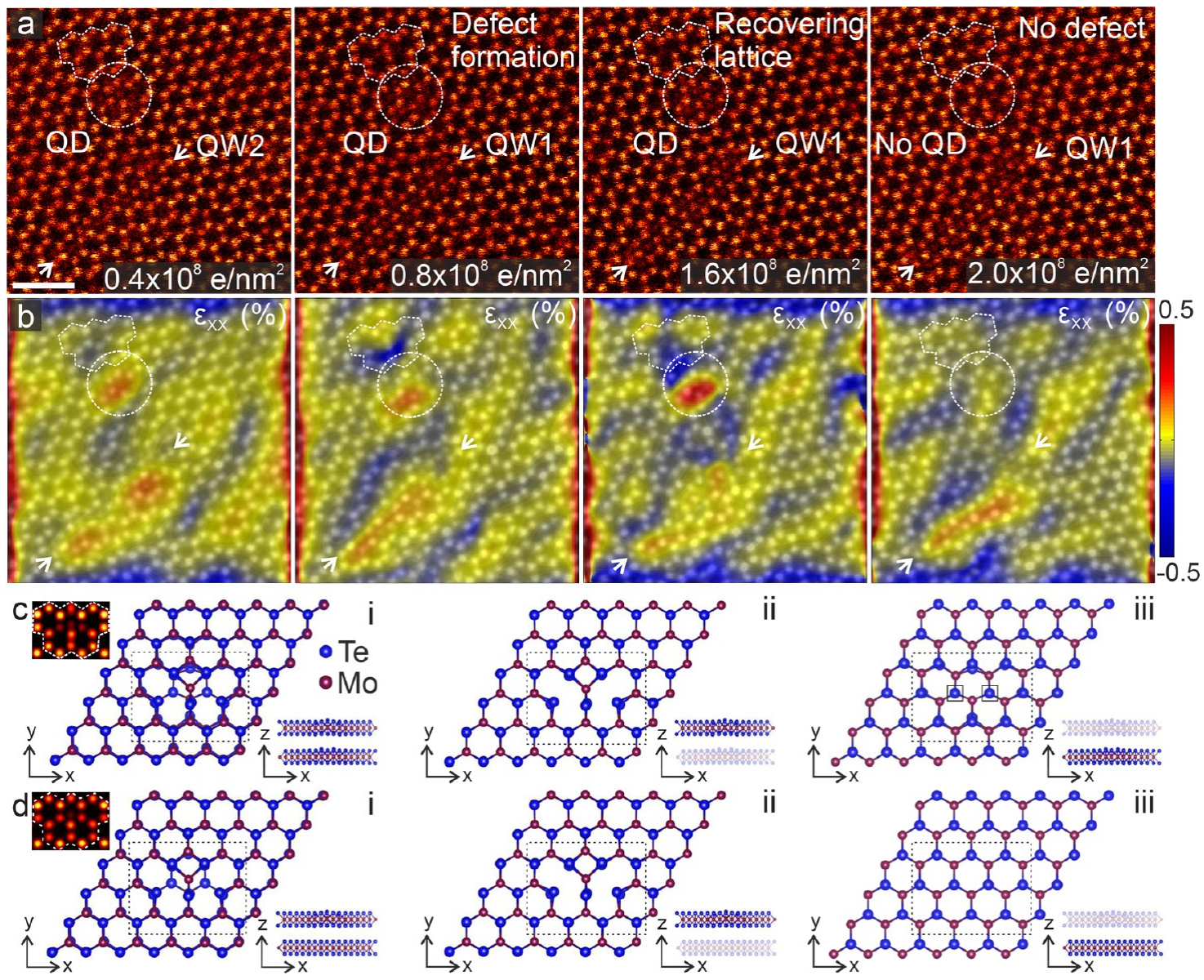

Figure 5. Dynamics of QDs and QWs under electron irradiation. (a) STEM-HAADF image sequence showing the change of the atomic structure of a QD and QW in encapsulated bilayer $\mathrm{MoTe}_{2}$ (background subtracted using a Gaussian blur of $20 \mathrm{px}$ ). The scale bar is $0.5 \mathrm{~nm}$. (b) $\varepsilon_{x x}$ strain maps corresponding to panel a. (c,d) Atomic models of the butterfly-like defect in the second frame of panel a. The areas marked by dashed black frames correspond to those shown in simulated images in the insets. The black squares show the location of $\mathrm{Te}$ vacancies at the second $\mathrm{MoTe}_{2}$ layer shown in panel c-iii.

presumably due to the negative strain (increasing blue area), the QW structure is transformed from a 2 -fold rotationsymmetric type (QW2) to a reflection-symmetric (QW1) type. Although the defect structure in the second frame is a bit unclear, it appears to involve one slightly displaced Mo atom, two missing Te, and one missing Mo, as shown in panel c-i. To further understand this structure, we created two possible atomic models, as shown in panels c,d. While the first layer in panel c-ii involves one shifted and one missing Mo atom, the second layer in panel c-iii has two missing Te atoms. The other model (panel d-i) has no missing Te atoms at the second layer. From the simulated images of both models (see insets of panels $c-i$ and $d-i)$, we conclude that the structure with two missing Te atoms at the second $\mathrm{MoTe}_{2}$ layer is in better agreement with the experimental image. In the third frame of panel a, the displaced Mo atom has moved back to its lattice position. Finally, in the last frame, both Mo and Te vacancies are filled, and at the same time the QD disappears. Another example of atomic dynamics at a QD is shown in Supplementary Figure 7.

Electronic Structure. Since both of the observed defect types are created within the semiconducting $1 \mathrm{H}$ phase, it is worth asking whether their electronic properties differ significantly from those of the host structure. The electronic band structures calculated for the $1 \mathrm{H}$ phase both in the primitive hexagonal 3-atom and orthorhombic 6-atom unit cells are shown in Figure 6a and d. The DFT band gap is ca. $1.14 \mathrm{eV}$. The unfolded electronic band structures for the QD (hexagonal supercell) and QWs (orthorhombic supercells) are shown in panels $b$, e, and $\mathrm{f}$. While the extra bands due to the QD defect resemble localized molecular states, in both the QW1 and QW2 defects there is clear evidence for metallic conduction in the direction of the defect line. This suggests the possibility of applications as conductive wires or nanoscale antennas.

Edge Structure. Finally, despite the overall stability of the encapsulated $\mathrm{MoTe}_{2}$ under the electron beam, its edges appear unstable. Figure $7 \mathrm{a}$ shows the atomic structure of an edge in bilayer $\mathrm{MoTe}_{2}$. Ideal symmetric edges would be terminated either by Te or Mo atoms. However, elemental identification at the edge is complicated by the liquid-like behavior of the structure during continuous imaging (see Supplementary Videos and Supplementary Figure 8). This is in contrast to the nonencapsulated structure, for which the atomic structure remains ordered (see Supplementary Figure 9). To elucidate on the disordered structure, we carried out molecular dynamics simulations of an encapsulated $\mathrm{MoTe}_{2}$ structure at room temperature. These simulations reveal a clear tendency for the $\mathrm{Te}$ atoms to detach from the $\mathrm{MoTe}_{2}$ crystal and spread between the graphene sheets. Interestingly, no electron-beam effect is required to produce this behavior. Due to the similarity between the experimental image (Figure $7 \mathrm{~b}$ ) and the simulated 

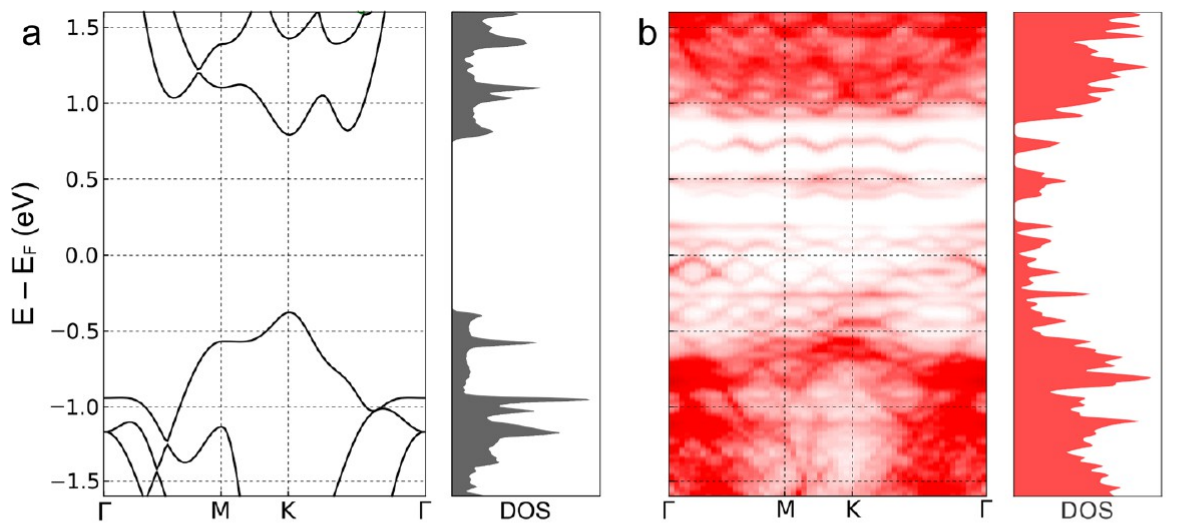

C
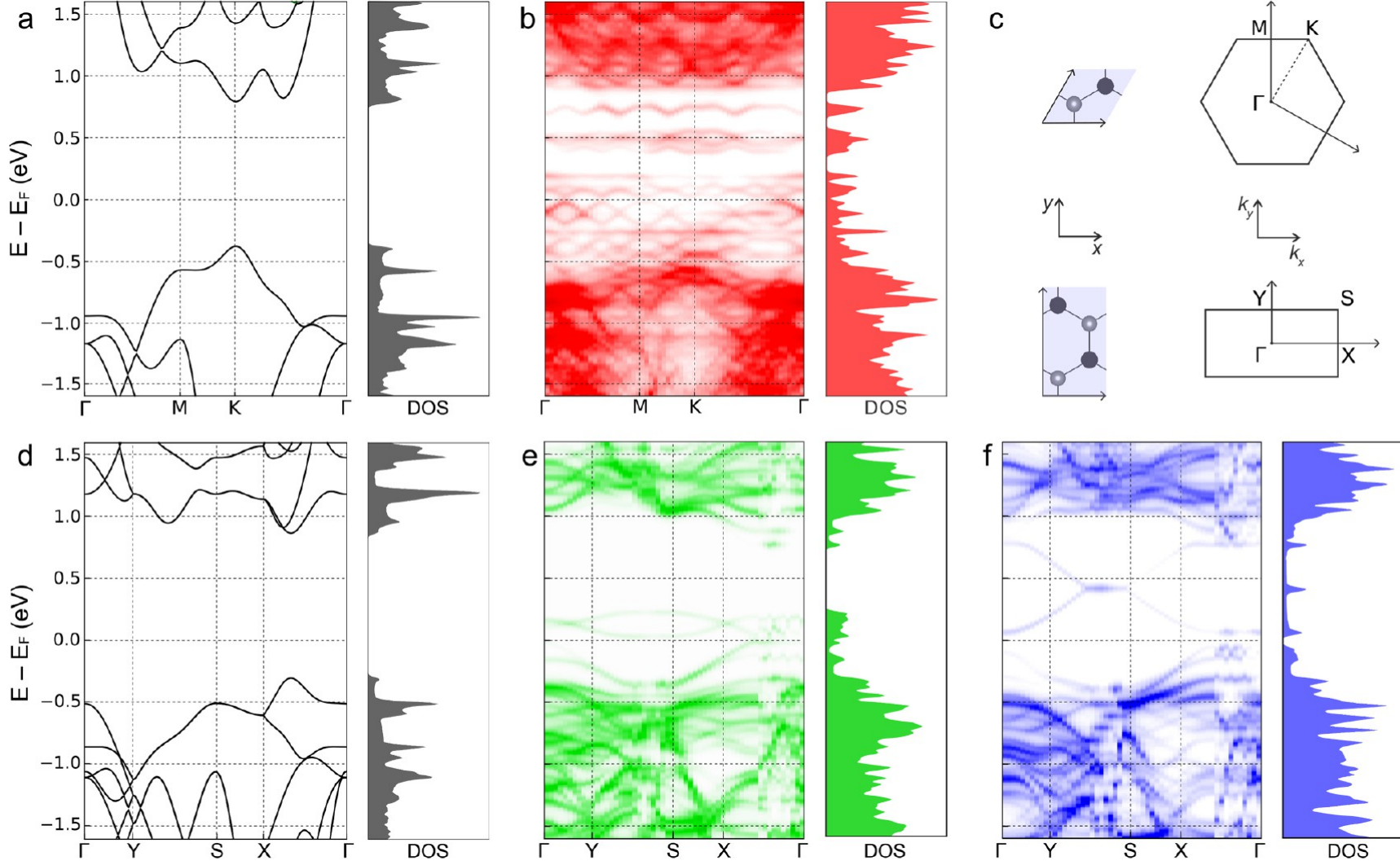

Figure 6. Unfolded electronic band structures calculated with DFT. The band structure (hexagonal cell) of the (a) $1 \mathrm{H}$ phase of monolayer MoTe $\mathrm{e}_{2}$ and (b) QD defect. (c) The lattice and the first Brillouin zone of the 3-atom and 6-atom unit cells. The electronic band structure (orthorhombic cell) of the (d) $1 \mathrm{H}$ phase of monolayer $\mathrm{MoTe}_{2}$, (e) QW1 defect, and (f) QW2 defect. The intensity of some of the bands (in panels b, e, and f) is weaker because these states are due to the localized defect in the unfolded band structure, and the segmentation of the lines reflect the finite k-point sampling.
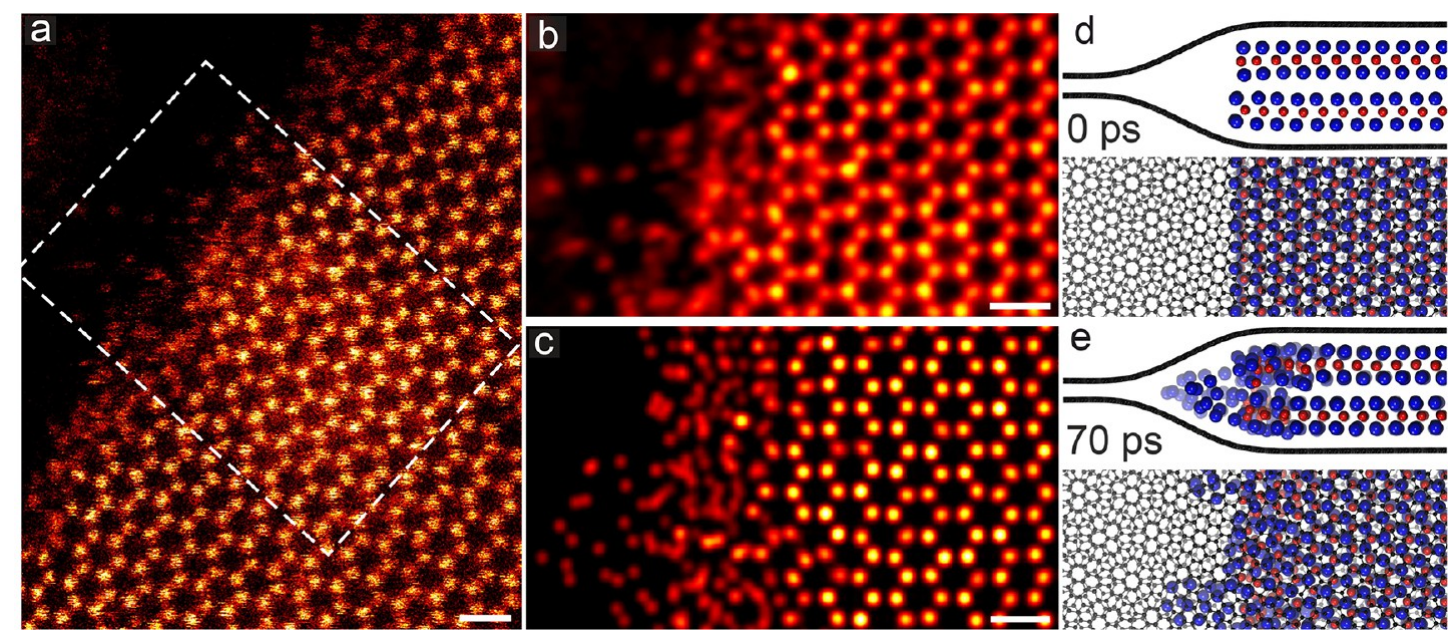

Figure 7. Edge structure of encapsulated bilayer $\mathrm{MoTe}_{2}$. (a) STEM-HAADF image showing the edge of graphene-encapsulated bilayer MoTe $e_{2}$. The image displays raw data in false color. (b) A close-up of the region indicated by the white dashed line in panel a. The image has been treated by a Gaussian blur with a radius of $4 \mathrm{px}$. (c) Simulated snapshot of graphene-encapsulated bilayer $\mathrm{MoTe}_{2}$ after $70 \mathrm{ps}$ at $300 \mathrm{~K}$. The scale bars are $0.5 \mathrm{~nm}$. (d, e) Side and top views of the model structure at the beginning of the simulation and after $70 \mathrm{ps}$ at $300 \mathrm{~K}$.

one (Figure $7 \mathrm{c}$ ) we believe that $\mathrm{Te}$ atoms are also responsible for the dynamics observed in the microscope. Following ref 34, we estimate the pressure due to graphene encapsulation to be in the order of a few GPa close to the edge of the $\mathrm{MoTe}_{2}$ structure. In contrast to our present results with $\mathrm{MoTe}_{2}$, graphene-encapsulated $\mathrm{MoS}_{2}$ has been reported to have an easily resolvable edge structure. ${ }^{27,35}$

\section{CONCLUSIONS}

Unprotected $\mathrm{MoTe}_{2}$ is highly susceptible to damage during TEM imaging, which leads to the creation of vacancies, especially at the edges of the suspended material, but also to interesting atomic-scale dynamics. For example, the semiconducting $1 \mathrm{H}$ phase can be locally turned into the semimetallic $1 \mathrm{~T}^{\prime}$ phase, which can in turn be transformed 
back to defective $2 \mathrm{H}$ due to continuing loss of atoms. Electron irradiation also leads to the migration of grain boundaries between $1 \mathrm{H}$ grains with a $60^{\circ}$ misorientation. Further, a 3-fold rotationally symmetric defect appears at high electron doses. All of these dynamics are significantly suppressed when $\mathrm{MoTe}_{2}$ is encapsulated between two graphene layers, which allows the study of its intrinsic defects. We observe a relatively high concentration $\left(0.58 / 100 \mathrm{~nm}^{2}\right)$ of $1 \mathrm{~nm}$-sized quantum dot structures, associated with the 3 -fold rotationally symmetric defect, as well as two different quantum wire structures (with a joint concentration of about $0.48 / 100 \mathrm{~nm}^{2}$ ): a reflectionsymmetric defect (QW1) and 2-fold rotationally symmetric defect (QW2). Both the possibility of introducing phase transitions between electrically very different phases and the existence of quantum dot and quantum wire defects with midgap states in the otherwise semiconducting material indicate a possibility for tuning the material properties through defect engineering. The amorphous nature of the edge in encapsulated $\mathrm{MoTe}_{2}$ can lead to transport of Te atoms through the edge, and thus a metallic Te ion channel surrounding the semiconducting $\mathrm{MoTe}_{2}$ can be obtained.

\section{METHODS}

Sample Preparation. The $\mathrm{MoTe}_{2}$ flakes were exfoliated on a $\mathrm{SiO}_{2} / \mathrm{Si}$ substrate and then transferred onto TEM grids with a carbonbased support membrane (QUANTIFOIL). Monolayer graphene sheets were synthesized by chemical vapor deposition in a mixture of $50 \mathrm{sccm} \mathrm{CH} \mathrm{CH}_{4}$ and $2000 \mathrm{sccm} \mathrm{Ar} / \mathrm{H}_{2}$ gases at $960{ }^{\circ} \mathrm{C}$. First, a graphene sheet was transferred from $\mathrm{Cu}$ foil onto a TEM grid without polymer, and then the $\mathrm{MoTe}_{2}$ flakes were transferred on top. The obtained $\mathrm{MoTe}_{2} /$ graphene stack was then transferred onto another graphene sheet on $\mathrm{Cu}$ foil. The $\mathrm{Cu}$ foils were etched with $\mathrm{FeCl}_{3}$.

Transmission Electron Microscopy. STEM images of $\mathrm{MoTe}_{2}$ and graphene-encapsulated $\mathrm{MoTe}_{2}$ were recorded using a Nion UltraSTEM 100 electron microscope operated at a $60 \mathrm{kV}$ accelerating voltage in near ultrahigh vacuum $\left(2 \times 10^{-7} \mathrm{~Pa}\right)$ using the high angle annular dark field (HAADF) detector with a collection angle of $80-$ $200 \mathrm{mrad}$. For TEM diffraction experiments (Supporting Information) we used a Delong instruments LVEM5 table-top transmission electron microscope operated at $5 \mathrm{kV}$.

DFT and STEM Image Simulations. Density functional theory (DFT) simulations were carried out using the grid-based projectoraugmented wave (GPAW) software package ${ }^{36}$ to study the properties of the unit cell and supercells of monolayer and bilayer $\mathrm{MoTe}_{2}$. For unit cells, we used a plane-wave basis (cutoff energy $700 \mathrm{eV}, 16 \times 16 \times$ $1 \mathrm{k}$-point mesh) to relax the atomic positions using the C09 van der Waals functional ${ }^{37}$ (the DF2 functional ${ }^{38}$ was additionally used for relative energies of different $\mathrm{MoTe}_{2}$ phases shown in Supplementary Table 1). The atomic structure of supercells of $\mathrm{MoTe}_{2}$ including QDs and QWs was relaxed with periodic boundary conditions in the finitedifference mode with the grid spacing of $0.2 \AA$ and a $3 \times 3 \times 1$ k-point mesh so that maximum forces were $<0.05 \mathrm{eV} \AA^{-1}$. A double- $\zeta$ linear combination of atomic orbitals basis was used to speed up the calculations for the larger simulated structures. STEM-HAADF and TEM diffraction simulations were performed using the QSTEM software with parameters corresponding to the experiments. ${ }^{39}$ The NEB simulation was performed with no frozen atoms and with five intermediate images. ${ }^{40}$ All optimized atomic structures are provided in the Supporting Information.

Molecular Dynamics. To study the atomic structure of $\mathrm{MoTe}_{2}$ edges encapsulated by graphene, we created a supercell with a size of $28 \times 58 \AA^{2}$ of bilayer $2 \mathrm{H} \mathrm{MoTe} \mathrm{H}_{2}$. The two graphene sheets are misoriented by $\sim 18^{\circ}$ with respect to each other, and the resulting moiré pattern periodicity is $\sim 14 \AA$. The reactive bond-orderdependent force field that supports bond breaking and bond formation was used to describe the interaction between molybdenum and tellurium atoms and also the interaction between the carbon atoms. ${ }^{41,42}$ Long distance van der Waals interactions between the carbon atoms and between $\mathrm{C}-\mathrm{Mo}$ and $\mathrm{C}-\mathrm{Te}$ were treated with Morse potentials. All calculations were performed with Large-scale Atomic/ Molecular Massively Parallel Simulator (LAMMPS) code. ${ }^{43,44}$ The total potential energy was minimized by relaxing both layers without applying any constraints until the forces were below $10^{-3} \mathrm{eV} / \AA$ and the strain on the whole structure was negligible (pressure below 1 bar). Later, the temperature was increased to $300 \mathrm{~K}$ for $1 \mathrm{~ns}$ for the molecular dynamics simulations (graphene sheets were kept at $0 \mathrm{~K}$ ).

\section{ASSOCIATED CONTENT}

\section{Supporting Information}

The Supporting Information is available free of charge on the ACS Publications website at DOI: 10.1021/acs.chemmater.7b03760.

TEM and electron diffraction images, STEM-HAADF images of monolayer, bilayer and encapsulated $\mathrm{MoTe}_{2}$, the atomic model of graphene-encapsulated $\mathrm{MoTe}_{2}$ monolayer, DFT total energies of $2 \mathrm{H}, 1 \mathrm{~T}$, and $1 \mathrm{~T}^{\prime}$ phases of $\mathrm{MoTe}_{2}$ monolayers and bilayers, formation energies of monolayer $\mathrm{MoTe}_{2}$ defects (PDF)

Atomic configuration files of the structures shown in the manuscript (ZIP)

Videos showing the edge of graphene-encapsulated $\mathrm{MoTe}_{2}$ bilayer (ZIP)

\section{AUTHOR INFORMATION}

\section{Corresponding Author}

*E-mail: jani.kotakoski@univie.ac.at.

ORCID

Kenan Elibol: 0000-0002-8765-2794

Toma Susi: 0000-0003-2513-573X

Giacomo Argentero: 0000-0003-0406-8208

Jannik C. Meyer: 0000-0003-4023-0778

Jani Kotakoski: 0000-0002-1301-5266

\section{Notes}

The authors declare no competing financial interest.

\section{ACKNOWLEDGMENTS}

K.E., M.R.A.M., and J.C.M. acknowledge support from the Austrian Science Fund (FWF) through project P25721-N20, T.S. acknowledges support through project P 28322-N36, and M.R.A.M. and J.K. acknowledge support through project I3181N36. T.J.P. was supported by the European Union's Horizon 2020 research and innovation programme under the Marie Skłodowska-Curie grant agreement No. 655760-DIGIPHASE. J.C.M. acknowledges funding by the European Research Council Grant No. 336453-PICOMAT, and J.K. acknowledges funding from the Wiener Wissenschafts-, Forschungs- und Technologiefonds (WWTF) via project MA14-009. We also acknowledge generous computational resources from the Vienna Scientific Cluster.

\section{REFERENCES}

(1) Susi, T.; Meyer, J. C.; Kotakoski, J. Manipulating low-dimensional materials down to the level of single atoms with electron irradiation. Ultramicroscopy 2017, 180, 163-172.

(2) Deng, K.; et al. Experimental observation of topological Fermi arcs in type-II Weyl semimetal $\mathrm{MoTe}_{2}$. Nat. Phys. 2016, 12, 11051110.

(3) Han, G. H.; Hoon Keum, D.; Zhao, J.; Gyu Shin, B.; Song, S.; Jun Bae, J.; Lee, J.; Ho Kim, J.; Kim, H.; Hee Moon, B.; Hee Lee, Y. 
Absorption dichroism of monolayer $1 \mathrm{~T}^{\prime}-\mathrm{MoTe}_{2}$ in visible range. $2 \mathrm{D}$ Mater. 2016, 3, 031010.

(4) Joshi, J.; Stone, I. R; Beams, R.; Krylyuk, S.; Kalish, I.; Davydov, A. V.; Vora, P. M. Phonon anharmonicity in bulk Td-MoTe 2 . Appl. Phys. Lett. 2016, 109, 031903.

(5) Diaz, H. C.; Chaghi, R.; Ma, Y.; Batzill, M. Molecular beam epitaxy of the Van der Waals heterostructure $\mathrm{MoTe}_{2}$ on $\mathrm{MoS}_{2}$ : phase, thermal, and chemical stability. 2D Mater. 2015, 2, 044010.

(6) Keum, D. H.; Cho, S.; Kim, J. H.; Choe, D.-H.; Sung, H.-J.; Kan, M.; Kang, H.; Hwang, J.-Y.; Kim, S. W.; Yang, H.; Chang, K. J.; Lee, Y. $\mathrm{H}$. Bandgap opening in few-layered monoclinic $\mathrm{MoTe}_{2}$. Nat. Phys. 2015, 11, 482-486.

(7) Kuiri, M.; Chakraborty, B.; Paul, A.; Das, S.; Sood, A. K.; Das, A. Enhancing photoresponsivity using $\mathrm{MoTe}_{2}$-graphene vertical heterostructures. Appl. Phys. Lett. 2016, 108, 063506.

(8) Park, J. C.; Yun, S. J.; Kim, H.; Park, J. H.; Chae, S. H.; An, S. J.; Kim, J. G.; Kim, S. M.; Kim, K. K.; Lee, Y. H. Phase-engineered synthesis of centimeter-scale $1 \mathrm{~T}$ - and $2 \mathrm{H}$-molybdenum ditelluride thin films. ACS Nano 2015, 9, 6548-6554.

(9) Duerloo, K.-A. N.; Li, Y.; Reed, E. J. Structural phase transitions in two-dimensional Mo- and W-dichalcogenide monolayers. Nat. Commun. 2014, 5, 4214.

(10) Sun, Y.; Wu, S. C.; Ali, M. N.; Felser, C.; Yan, B. Prediction of Weyl semimetal in orthorhombic $\mathrm{MoTe}_{2}$. Phys. Rev. B: Condens. Matter Mater. Phys. 2015, 92, 161107.

(11) Li, Y.; Duerloo, K.-A. N.; Wauson, K.; Reed, E. J. Structural semiconductor-to-semimetal phase transition in two-dimensional materials induced by electrostatic gating. Nat. Commun. 2016, 7, 10671.

(12) Huang, H. H.; Fan, X.; Singh, D. J.; Chen, H.; Jiang, Q.; Zheng, W. T. Controlling phase transition for single-layer $\mathrm{MTe}_{2}(\mathrm{M}=\mathrm{Mo}$ and $\mathrm{W})$ : modulation of the potential barrier under strain. Phys. Chem. Chem. Phys. 2016, 18, 4086-4094.

(13) Cho, S.; Kim, S.; Kim, J. H.; Zhao, J.; Seok, J.; Keum, D. H.; Baik, J.; Choe, D.-h.; Chang, K. J.; Suenaga, K.; Kim, S. W.; Lee, Y. H.; Yang, $\mathrm{H}$. Phase patterning for ohmic homojunction contact in $\mathrm{MoTe}_{2}$. Science 2015, 349, 625-628.

(14) Song, S.; Keum, D. H.; Cho, S.; Perello, D.; Kim, Y.; Lee, Y. H. Room Temperature Semiconductor-Metal Transition of $\mathrm{MoTe}_{2}$ Thin Films Engineered by Strain. Nano Lett. 2016, 16, 188-193.

(15) Zhang, K.; Bao, C.; Gu, Q.; Ren, X.; Zhang, H.; Deng, K.; Wu, Y.; Li, Y.; Feng, J.; Zhou, S. Raman signatures of inversion symmetry breaking and structural phase transition in type-II Weyl semimetal $\mathrm{MoTe}_{2}$. Nat. Commun. 2016, 7, 13552.

(16) Lin, Z.; Carvalho, B. R.; Kahn, E.; Lv, R.; Rao, R.; Terrones, H.; Pimenta, M. A.; Terrones, M. Defect engineering of two-dimensional transition metal dichalcogenides. 2D Mater. 2016, 3, 022002.

(17) Lin, Y.-C.; Björkman, T.; Komsa, H.-P.; Teng, P.-Y.; Yeh, C.-H.; Huang, F.-S.; Lin, K.-H.; Jadczak, J.; Huang, Y.-S.; Chiu, P.-W.; Krasheninnikov, A. V.; Suenaga, K. Three-fold rotational defects in two-dimensional transition metal dichalcogenides. Nat. Commun. 2015, 6, 6736.

(18) Enyashin, A. N.; Bar-sadan, M.; Houben, L.; Seifert, G. Line defects in molybdenum disulfide layers. J. Phys. Chem. C 2013, 117, 10842-10848.

(19) Ghorbani-Asl, M.; Enyashin, A. N.; Kuc, A.; Seifert, G.; Heine, T. Defect-induced conductivity anisotropy in $\mathrm{MoS}_{2}$ monolayers. Phys. Rev. B: Condens. Matter Mater. Phys. 2013, 88, 245440.

(20) van der Zande, A. M.; Huang, P. Y.; Chenet, D. A.; Berkelbach, T. C.; You, Y.; Lee, G.-H.; Heinz, T. F.; Reichman, D. R.; Muller, D. A.; Hone, J. C. Grains and grain boundaries in highly crystalline monolayer molybdenum disulphide. Nat. Mater. 2013, 12, 554-561.

(21) Dumcenco, D.; Ovchinnikov, D.; Lopez Sanchez, O.; Gillet, P.; Alexander, D. T. L.; Lazar, S.; Radenovic, A.; Kis, A. Large-area $\mathrm{MoS}_{2}$ grown using $\mathrm{H}_{2} \mathrm{~S}$ as the sulfur source. $2 D$ Mater. 2015, 2, 044005.

(22) Ma, Y.; Kolekar, S.; Coy Diaz, H.; Aprojanz, J.; Miccoli, I.; Tegenkamp, C.; Batzill, M. Metallic twin grain boundaries embedded in $\mathrm{MoSe}_{2}$ monolayers grown by molecular beam epitaxy. ACS Nano 2017, 11, 5130-5139.
(23) Diaz, H. C.; Ma, Y.; Chaghi, R.; Batzill, M. High density of (pseudo) periodic twin-grain boundaries in molecular beam epitaxygrown van der Waals heterostructure: $\mathrm{MoTe}_{2} / \mathrm{MoS}_{2}$. Appl. Phys. Lett. 2016, 108, 191606.

(24) Zhou, W.; Zou, X.; Najmaei, S.; Liu, Z.; Shi, Y.; Kong, J.; Lou, J.; Ajayan, P. M.; Yakobson, B. I.; Idrobo, J.-C. Intrinsic Structural Defects in Monolayer Molybdenum Disulfide. Nano Lett. 2013, 13, 26152622.

(25) Wang, Q. H.; Kalantar-Zadeh, K.; Kis, A.; Coleman, J. N.; Strano, M. S. Electronics and optoelectronics of two-dimensional transition metal dichalcogenides. Nat. Nanotechnol. 2012, 7, 699-712.

(26) Zhang, Z.; Zou, X.; Crespi, V. H.; Yakobson, B. I. Intrinsic magnetism of grain boundaries in two-dimensional metal dichalcogenides. ACS Nano 2013, 7, 10475-10481.

(27) Zan, R.; Ramasse, Q. M.; Jalil, R.; Georgiou, T.; Bangert, U.; Novoselov, K. S. Control of radiation damage in $\mathrm{MoS}_{2}$ by graphene encapsulation. ACS Nano 2013, 7, 10167-10174.

(28) Nguyen, L.; Komsa, H.-P.; Khestanova, E.; Kashtiban, R. J.; Peters, J. J. P.; Lawlor, S.; Sanchez, A. M.; Sloan, J.; Gorbachev, R. V.; Grigorieva, I. V.; Krasheninnikov, A. V.; Haigh, S. J. Atomic defects and doping of monolayer $\mathrm{NbSe}_{2}$. ACS Nano 2017, 11, 2894-2904.

(29) Iberi, V.; Liang, L.; Ievlev, A. V.; Stanford, M. G.; Lin, M.-W.; Li, X.; Mahjouri-Samani, M.; Jesse, S.; Sumpter, B. G.; Kalinin, S. V.; Joy, D. C.; Xiao, K.; Belianinov, A.; Ovchinnikova, O. S. Nanoforging single layer $\mathrm{MoSe}_{2}$ through defect engineering with focused helium ion beams. Sci. Rep. 2016, 6, 30481.

(30) Hong, J.; Wang, Y.; Wang, A.; Lv, D.; Jin, C.; Xu, Z.; Probert, M. I. J.; Yuan, J.; Zhang, Z. Atomistic dynamics of sulfur-deficient highsymmetry grain boundaries in molybdenum disulfide. Nanoscale 2017, 9, 10312-10320.

(31) Lin, J.; Pantelides, S. T.; Zhou, W. Vacancy-Induced Formation and Growth of Inversion Domains in Transition-Metal Dichalcogenide Monolayer. ACS Nano 2015, 9, 5189-5197.

(32) Azizi, A.; Zou, X.; Ercius, P.; Zhang, Z.; Elías, A. L.; PereaLópez, N.; Stone, G.; Terrones, M.; Yakobson, B. I.; Alem, N. Dislocation motion and grain boundary migration in two-dimensional tungsten disulphide. Nat. Commun. 2014, 5, 4867.

(33) Zhao, J.; Nam, H.; Ly, T. H.; Yun, S. J.; Kim, S.; Cho, S.; Yang, H.; Lee, Y. H. Chain Vacancies in 2D Crystals. Small 2017, 13, 1601930.

(34) Vasu, K. S.; Prestat, E.; Abraham, J.; Dix, J.; Kashtiban, R. J.; Beheshtian, J.; Sloan, J.; Carbone, P.; Neek-Amal, M.; Haigh, S. J.; Geim, A. K.; Nair, R. R. Van der Waals pressure and its effect on trapped interlayer molecules. Nat. Commun. 2016, 7, 12168.

(35) Algara-Siller, G.; Kurasch, S.; Sedighi, M.; Lehtinen, O.; Kaiser, $\mathrm{U}$. The pristine atomic structure of $\mathrm{MoS}_{2}$ monolayer protected from electron radiation damage by graphene. Appl. Phys. Lett. 2013, 103, 203107.

(36) Mortensen, J. J.; Hansen, L. B.; Jacobsen, K. W. Real-space grid implementation of the projector augmented wave method. Phys. Rev. B: Condens. Matter Mater. Phys. 2005, 71, 035109.

(37) Cooper, V. R. Van der Waals density functional: An appropriate exchange functional. Phys. Rev. B: Condens. Matter Mater. Phys. 2010 $81,161104$.

(38) Lee, K.; Murray, É. D.; Kong, L.; Lundqvist, B. I.; Langreth, D. C. Higher-accuracy van der Waals density functional. Phys. Rev. B: Condens. Matter Mater. Phys. 2010, 82, 081101.

(39) Koch, C. Determinations of core structure periodicity and point defect density along dislocations, Dissertation, 2002.

(40) Henkelman, G.; Jonsson, H. Improved tangent estimate in the nudged elastic band method for finding minimum energy paths and saddle points. J. Chem. Phys. 2000, 113, 9978-9985.

(41) Aktulga, H.; Fogarty, J.; Pandit, S.; Grama, A. Parallel reactive molecular dynamics: Numerical methods and algorithmic techniques. Parallel Comput. 2012, 38, 245-259.

(42) Onofrio, N.; Guzman, D.; Strachan, A. The dynamics of copper intercalated molybdenum ditelluride. J. Chem. Phys. 2016, 145, 194702. 
(43) Plimpton, S. Fast Parallel Algorithms for Short-Range Molecular Dynamics. J. Comput. Phys. 1995, 117, 1-19.

(44) Plimpton, S. J.; Thompson, A. P. Computational aspects of many-body potentials. MRS Bull. 2012, 37, 513-521. 